\title{
$d$-Dimensional Reverse Euclidean Distance Transformation and Euclidean Medial Axis Extraction in Optimal Time
}

\author{
David Coeurjolly \\ Laboratoire LIRIS \\ Université Lumière Lyon 2 \\ 5 avenue Pierre Mendès-France \\ F-69676 Bron, France \\ dcoeurjo@univ-lyon2.fr
}

\begin{abstract}
In this paper, we present optimal in time algorithms to solve the reverse Euclidean distance transformation and the reversible medial axis extraction problems for $d$-dimensional images. In comparison to previous technics, the proposed Euclidean medial axis may contain less points than the classical medial axis.
\end{abstract}

Keywords: Reverse Euclidean distance transform, medial axis extraction, $d$-dimensional shapes.

\section{Introduction}

In binary images, the distance transformation (DT) and the geometrical skeleton extraction are classical tools for shape analysis $[15,16]$. The distance transformation consists in labelling each pixel of an object with the distance to the closest pixel of its complement (also called the background). Obviously, a distance transformation algorithm is deeply linked to the subjacent metric. In the digital image literature, the problem of approximating the Euclidean distance or isotropic property of digital object is worth interesting to. Hence, for the DT problem, we have mask based or chamfer distances $[2,14,16,19]$; vector displacement based Euclidean distance [5, 12]; Voronoi diagram based Euclidean distance $[4,8]$ or Squared distance based Euclidean distance $[7,9,17]$. In a computational cost point of view, several of these methods lead to optimal in time algorithms in order to compute the error-free Euclidean Distance Transform (EDT) for $n$-dimensional binary images $[4,7,8]$.

Skeleton or medial axis is a classical and convenient representation of a shape for description or recognition purpose [1]. Many definitions exist to define such an object [10]. A classical one defines the skeleton as the set of center pixels of maximal disks covering the shapes. A maximal disk is a disk contained in the shape not exactly covered by another disk contained in the shape. Many discrete implementations of these models have been proposed either for chamfer distances $[2,6,15]$ or for Euclidean distance $[13,18,19]$. 
In this paper, we investigate the $d$-dimensional medial axis (MA for short) extraction upon the error-free Euclidean distance. A first problem prior to the MA extraction is the reverse Euclidean distance transformation (REDT). Formally, given a set of points associated to their Euclidean distance values, how can we reconstruct the shape resulting from the overlapping of the corresponding balls ? An optimal in time algorithm is given to solve this problem. Based on this process, we present an optimal in time algorithm to compute a reduced medial axis on $d$-dimensional shapes.

In section 2, we first detail algorithms to solve the EDT problem for $d$ dimension in a linear time. Based on these technics, we optimize the REDT algorithm proposed by Satio and Toriwaki [18] to obtain a linear in time algorithm in the section 3. Then, in section 4, we present an optimal in time algorithm that extract a reversible subset of the classical medial axis. Finally, we present possible generalizations of these algorithms to other grids.

\section{$2 d$-Dimensional Euclidean Distance Transformation}

In [17], Saito et al. propose an $n$-dimensional approach for the EDT problem. The authors present a simple $n$-dimensional algorithm that labels image pixels with the squared distance to the closest background pixel. This process is done dimension by dimension and allows simple generalization in $d$-dimension.

We present the algorithm in the 2-D case: we consider a two dimensional binary image $P$ of size $n \times n, B$ denotes the non-empty set of background pixels and the output of the algorithm is a 2-D image $S=\left\{s_{i j}\right\}$ storing the squared distance transform. For each point $p(i, j)$ of the image, the squared distance transform is given by:

$$
\begin{aligned}
s_{p} & =\min _{q \in B}\left\{\operatorname{dist}^{2}(p, q)\right\} \\
& =\min _{q(x, y) \in B}\left\{(i-x)^{2}+(j-y)^{2}\right\} .
\end{aligned}
$$

This formulation of the problem leads to an efficient two pass process for the squared distance transform (SDT for short) labelling in 2-D:

1. Build from the source image $P$, an one-dimensional SDT according to the first dimension ( $x$-axis), denoted by $G=\left\{g_{i j}\right\}$, where

$$
g_{i j}=\min _{p(x, y) \in B}\left\{(i-x)^{2}\right\} .
$$

2. Then, construct the $s_{i j}$ image with a $y$-axis process:

$$
s_{i j}=\min _{y}\left\{g_{i y}+(j-y)^{2}, 1 \leq y \leq n\right\} .
$$

This algorithm provides a direct implementation of $d$-dimensional EDT algorithm: we only have to compute an one-dimensional SDT for the initialization step (step 1 of the previous algorithm) and then add, for each greater dimension, 

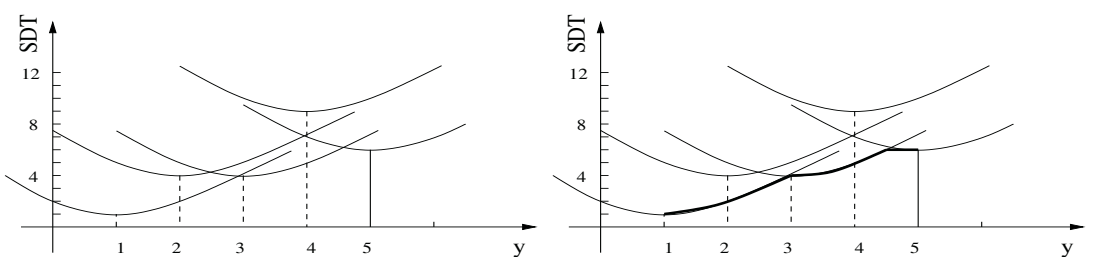

Fig. 1. Illustration of the Hirata's optimization: let $[1,4,4,9,4]$ be a column of $G$ after step 1, (left) the set of parabolas $(j-y)^{2}+g_{i y}$ and (right) the bold curve is the lower envelope. Thus the result of the minimization process is $[1,2,4,5,4]$.

a mix process (step 2) that merges results of inferior dimensions. In a computational cost point of view and given a $d$-dimensional binary shape of size $n^{d}$, the first step can be done in a linear time in the number of grid points, i.e. $O\left(n^{d}\right)$. In [17], the authors present an $O\left(A v g . n^{d}\right)$ algorithm that computes each mix step where $A v g$ denotes the average of the Euclidean distance values in the image $(A v g=O(n)$ without any assumptions on the input image). In [7] and [9], Hirata and Roerdnik et al. independently present optimal algorithms to solve the min operation and thus propose an optimal in time algorithm for the EDT. The idea is to see the min operation as a lower envelope computation of a set of parabolas. More precisely, let us suppose we have computed the step 1 of the algorithm $\left(x\right.$-axis SDT) and let $\left\{g_{i y}\right\}(1 \leq y \leq n)$ be a column of $G$. If we consider the set of parabolas $f_{y}^{i}(j)=(j-y)^{2}+g_{i y}$, the column $\left\{s_{i y}\right\}$ after step 2 is exactly the lower envelope of $f_{y}^{i}$ with $1 \leq y \leq n$ (see figure 1 ). In [7] and [9], the authors present an $O(n)$ algorithm to compute such a lower envelope using a parabola elimination process. Finally, for a $d$-dimensional image, the dimensional mix processes are computed in $O\left(n^{d}\right)$ and thus, the global cost to compute the EDT based on this approach is $O\left(n^{d}\right)$.

\section{Reverse Euclidean Distance Transformation}

\subsection{Definitions}

In [18], Saito and Toriwaki present both definitions and algorithms to compute the $d$-dimensional REDT. Using their notations and the dimension 2, let us consider $L$ as a set of $l$ points $\left\{\left(x_{m}, y_{m}\right)\right\}_{1 \leq m \leq l}$ and $f_{x_{m} y_{m}}$ the squared Euclidean distance value associated to the pixel $\left(x_{m}, y_{m}\right)$. The Reverse Euclidean Distance Transformation of $L$ consists in obtaining the set of points $P$ such that

$$
P=\left\{(i, j) \mid \exists m,\left(i-x_{m}\right)^{2}+\left(j-y_{m}\right)^{2}<f_{x_{m} y_{m}},\left(x_{m}, y_{m}\right) \in L\right\} .
$$

In other words, a point $(i, j)$ belongs to $P$ if it belongs to at least one disk whose center is a point $m$ of $L$, with radius $\sqrt{f_{x_{m} y_{m}}}$.

Let $F=\left\{f_{i j}\right\}$ be a picture of size $n \times n$ such that $f_{i j}$ is set to $f_{x_{m} y_{m}}$ if $(i, j)$ belongs to $L$ and 0 otherwise. The authors show that equation (5) is equivalent to 


$$
P=\left\{(i, j) \mid \max _{(x, y) \in F}\left\{f_{x y}-(i-x)^{2}-(j-y)^{2}\right\}>0\right\} .
$$

Hence, if we compute the map $H=\left\{h_{i j}\right\}$ such that

$$
h_{i j}=\max _{(x, y) \in F}\left\{f_{x y}-(i-x)^{2}-(j-y)^{2}\right\} .
$$

we obtain $P$ by extracting from $H$ all pixels of positive values. So, to build $H$ from $F$, we can decompose the computation into two one-dimensional processes:

1. Build from the image $F$ the picture $G=\left\{g_{i j}\right\}$ such that

$$
g_{i j}=\max _{x}\left\{f_{x j}-(i-x)^{2}, 1 \leq x \leq n\right\} .
$$

2. Build from $G$ the picture $H$ such that

$$
h_{i j}=\max _{y}\left\{g_{i y}-(j-y)^{2}, 1 \leq y \leq n\right\} .
$$

To prove this decomposition, we can substitute the equation (8) to the equation (9) and we obtain the equation (7). Finally, we can design similar algorithms to solve the REDT as those proposed for the EDT labelling; we only have to replace the minimization steps by the maximization steps and thus to compute upper envelope of parabolas (see next section). Note that this process can be easily extended to $d$-dimensional images, we just have to compute $d$ one-dimensional maximization steps. In [18], Saito and Toriwaki use their algorithm presented in [17] to compute the REDT and they obtain a computational cost in $O\left(A v g . n^{d}\right)$ for a $d$-dimensional image. In the next section, we detail an $O\left(n^{d}\right)$ algorithm to compute the REDT.

\subsection{Optimal REDT Algorithm}

The basic idea of the optimal REDT algorithm is to use the Hirata's parabola elimination process to compute maximization steps. We detail the optimization of step 1 in the previous algorithm, all other steps can be easily deduced. First of all, for a given column $j$ of $F$, we define a function describing a parabola:

$$
\mathcal{F}_{x}(i)=f_{x j}-(i-x)^{2}
$$

and a function that computes the abscissa of the intersection between two parabolas. Thus, we have to find the point $i$ such that $\mathcal{F}_{u}(i) \geq \mathcal{F}_{v}(i)$ with $u<v$. Hence, the "separation" between the parabolas is given by

$$
\mathcal{S} e p(u, v)=\left\lfloor\frac{u^{2}-v^{2}-f_{u j}+f_{v j}}{2(u-v)}\right\rfloor .
$$

Based on these elementary functions, the algorithm presented in figure 2 computes the upper envelope of the parabolas $\left\{\mathcal{F}_{x}\right\}$. This algorithm is derived from Meijster et al.'s one [9] (similar to Hirata's one). The idea is to manipulate 

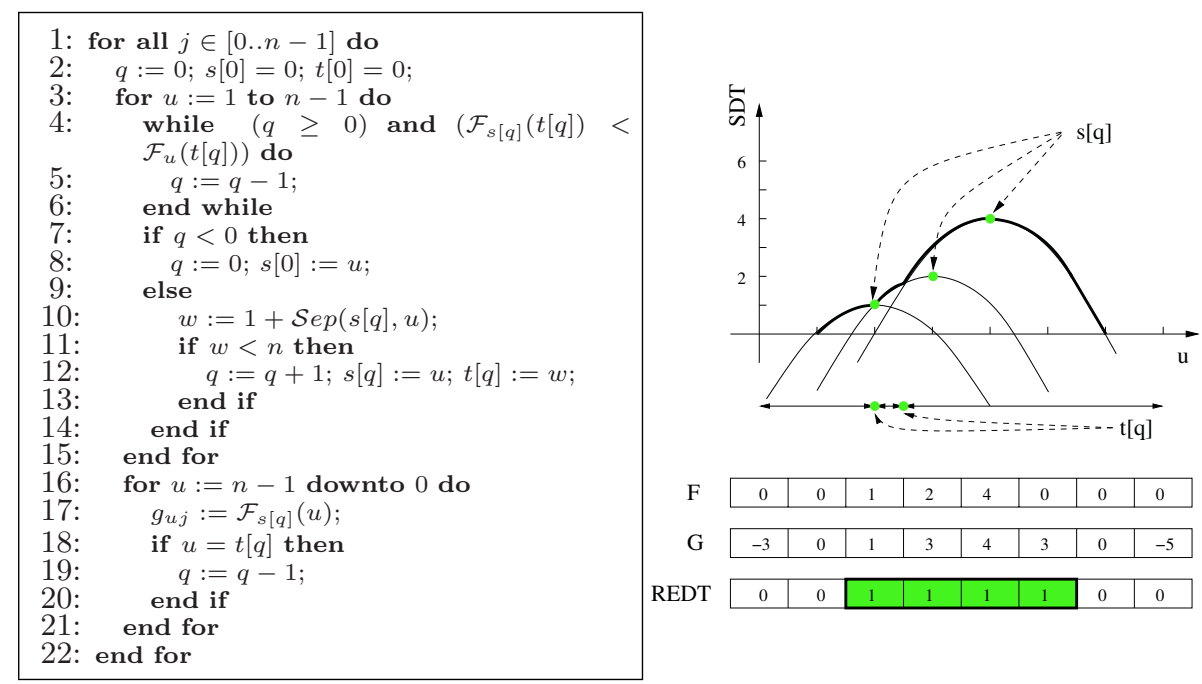

Fig. 2. Pseudo-code of the optimal one-dimensional upper envelope parabola computation (left); an illustration of the notations and an example in one-dimension (right).

two arrays $s$ and $t$ that simulate a parabola stack. The array $s$ contains the set of parabola apexes (tops of a parabola) of the upper envelope and $t$ the intersection abscissa between two consecutive parabolas in $s$. In lines $3-15$ we compute the upper envelope and those arrays $s$ and $t$; and in lines $16-21$, we construct the map $G$ using $s$ and $t$. The computational cost of this upper envelope extraction is $O(n)$ if $n$ is the size of a row in $F$. Finally, we can use this algorithm to compute step 2 and construct $P$, by thresholding $H$, in $O\left(n^{2}\right)$ if $F$ is a $n \times n$ image. More generally, if we apply it for all one-dimensional maximization steps, we have a global complexity in $O\left(n^{d}\right)$ for a $d$-dimensional image, which is optimal in time.

\section{Euclidean Medial Axis Extraction}

\subsection{Definitions}

First of all, we introduce some definitions.

Definition 1 (Maximal ball). A maximal ball is a ball contained in the shape not exactly covered by another ball contained in the shape.

Based on this property, we can define the medial axis:

Definition 2 (Medial axis). The medial axis (MA for short) of a shape is the set of maximal ball centers contained in the shape.

In [18], Saito and Toriwaki define a geometrical Euclidean skeleton based on elliptic paraboloids in dimension 2. Such an elliptic paraboloid of center $(i, j)$ and height $q_{i j}$ is given by the following equation 


$$
0 \leq z<q_{i j}-(x-i)^{2}-(y-j)^{2}
$$

The intersection between such a domain and the plane $z=0$ is a disk of center $(i, j)$ and radius $\sqrt{q_{i j}}$. We say that an elliptic paraboloid is contained in a shape $S$ if the disk of center $(i, j)$ and radius $\sqrt{q_{i j}}$ is contained in $S$. In the following, we prove that the Saito and Toriwaki's skeleton is a subset of the medial axis of a shape.

Definition 3 (Maximal elliptic paraboloid). A maximal elliptic paraboloid is an elliptic paraboloid contained in the shape not exactly covered by another elliptic paraboloid contained in the shape.

Note that this object can be generalized to $d$-dimension shapes.

Proposition 1. Let $(i, j)$ be a point in a continuous shape and $q_{i j}$ be a number. The disk $D$ of center $(i, j)$ and radius $\sqrt{q_{i j}}$ is maximal if and only if the elliptic paraboloid $P$ of center $(i, j)$ and height $q_{i j}$ is maximal.

Proof. Note that $D$ is the intersection between $P$ and $z=0$. We first prove the left to right implication. If we suppose that $P$ is not maximal, there exists another elliptic paraboloid $P^{\prime}$ such that $P^{\prime}$ contains $P$. Thus the intersection $D^{\prime}$ between $P^{\prime}$ and the plane $z=0$ contains the intersection $D$ between $P$ and the same plane. Hence, there exists a disk $D^{\prime \prime}$ contained in the shape that contains $D$, and so $D$ is not maximal.

Conversely, we suppose that $D$ is not maximal. Hence, there exists a disk $D^{\prime \prime}$ such that $D^{\prime \prime}$ contains $D$. We denote by $P^{\prime \prime}$ the elliptic paraboloid, uniquely defined, such that $D^{\prime \prime}$ is the intersection between $P^{\prime \prime}$ and $z=0$. If we suppose that $P^{\prime \prime}$ does not contain $P$, there exists a point $p \in P$ such that $p \notin P^{\prime \prime}$. Let us consider the intersections between $P$ and $P^{\prime \prime}$ with a plane $H$ perpendicular to $z=0$ that contains $p$ and the center of $P^{\prime \prime}$. In the plane $H$ and using the elliptic paraboloid definition, $P$ (resp. $P^{\prime \prime}$ ) leads to the domain

$$
0 \leq z<f_{u}-(x-u)^{2} \quad\left(\text { resp. } \quad 0 \leq z<f_{v}-(x-v)^{2}\right)
$$

with $u, v, f_{u}, f_{v} \in \mathbb{R}$ (see figure 3 - $(b)$ ). Since $H$ contains the center of $P^{\prime \prime}$ and $p$, these domains are not empty. Using the notations of figure $3-(b), D^{\prime \prime}$ contains $D$ implies that both $m$ and $n$ belong to $D^{\prime \prime}$. Furthermore, since $p$ does not belong to $P^{\prime \prime}$, the two parabolas given by equation 13 must have two intersection points $a$ and $b$. However, using the equation 13, such parabolas only have one intersection point if $u \neq v$. Since the upper parts of the parabolas are excluded, $u=v$ implies that the intersection is empty. Hence such a point $p$ does not exist and thus $P^{\prime \prime}$ contains $P$ which finally proves that $P$ is not maximal. Note that this proof can be generalized to other dimensions because we transform the problem into a 1-dimension parabola intersection.

Hence, in the continuous plane, maximal balls and maximal elliptic paraboloids coincide. In [18], the authors use the term "skeleton" to describe a geometric 


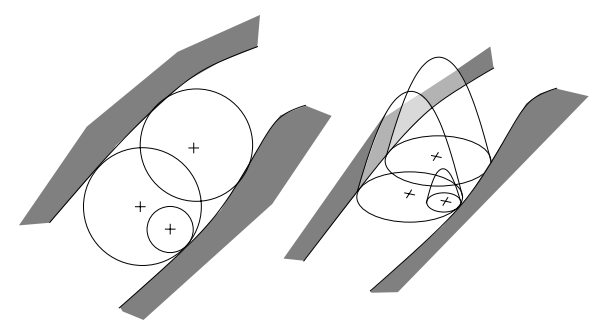

(a)

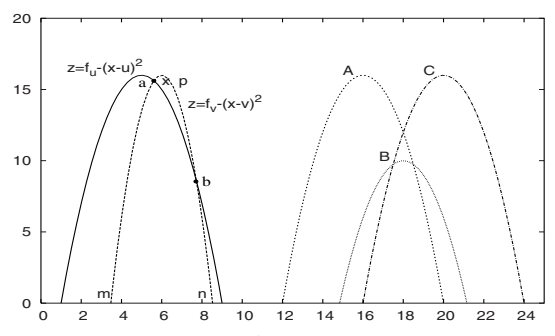

(b)

Fig. 3. Skeleton definitions: (a) comparison between maximal balls and maximal elliptic paraboloids; $(b)$ notations for the proof of the proposition 1 (left two parabolas) and differences between the medial axis and $S k$ (right three parabolas).

object not based on a topological feature preservation. Let $Q=\left\{q_{i j}\right\}$ be a SDT of the shape. The skeleton $S k$ is defined by

$$
\begin{aligned}
S k=\{(i, j) \mid & \exists(x, y),(i-x)^{2}+(j-y)^{2}<q_{i j}, \\
& \text { and } \left.\max _{(u, v)}\left\{q_{u v}-(x-u)^{2}-(y-v)^{2}\right\}=q_{i j}-(x-i)^{2}-(y-j)^{2}\right\} .
\end{aligned}
$$

In other words, $S k$ is the set of elliptic paraboloids that belong to the upper envelope (in dimension 2) of all elliptic paraboloids whose heights are given by the squared distance transform. Using proposition 1, we have the corollary:

Corollary 1. In the continuous plane, Sk is a subset of the medial axis. Furthermore, the original figure can be reconstructed by Sk.

Proof. First of all, all elliptic paraboloids that belong to the upper envelope are maximal by definition of such an envelope. Since maximal elliptic paraboloids and maximal balls coincide, points in $S k$ belong to the medial axis. Some maximal elliptic paraboloids may not belong to $S k$ as illustrated in figure $3-(b)$ in the 1-D case: the parabolas $\{A, B, C\}$ belong to the medial axis whereas only the parabolas $A$ and $C$ belong to $S k$ ( $B$ is covered by the union of $A$ and $C$ ). To prove the second statement, we remark that the definition of $S k$ strictly coincides with the reverse distance transformation equations of section 3 . Once $S k$ is computed, if we threshold the height values of the upper envelope elliptic paraboloids by 0 , we obtain the original shape [18].

If we consider a binary shape in dimension $d$, Saito and Toriwaki [18] use the $O\left(A v g . n^{d}\right)$ REDT process to extract the skeleton $S k$. The idea is to mark upper envelope elliptic paraboloids. If we use the optimal REDT algorithm proposed in the previous section, we obtain an algorithm to compute $S k$ in $O\left(n^{d}\right)$ which is optimal for the problem.

\subsection{Reduced Medial Axis Extraction}

In the previous section, we prove that the skeleton $S k$ is a subset of the medial axis in the continuous case. As illustrated in dimension 2 in figure 4-(left), this 
property does not hold in the discrete case. In the following we present a filtering process to transform $S k$ points into maximal ball centers in the discrete case. In the 2D case, let us consider a binary shape and its skeleton $S k$. We denote by $\left\{\mathcal{F}_{x}(i)\right\}_{i=0 . . N}$ the sequence of parabolas given by the intersection between the $S k$ elliptic paraboloids and the column $j$ of the image. Hence, each parabola is such that $\mathcal{F}_{x}(i)=f_{x j}-(i-x)^{2}$. In this one-dimension case, the differences between $S k$ and the discrete medial axis are illustrated in figure 4-(left): $\{D, E\}$ belong to $S k$ whereas only $D$ belongs to the medial axis.

We denote by $\mathcal{D}_{x}$ the disk associated to $\mathcal{F}_{x}(i)$ (i.e. a segment in the onedimensional case). Furthermore, we consider the discrete disk $D_{x}$ associated to $\mathcal{D}_{x}$ as the set of discrete points contained in $\mathcal{D}_{x}$. To only consider discrete maximal disk in $S k$, we have to remove all points $x$ such that $D_{x}$ is not maximal. Given two parabolas of centers $x$ and $x^{\prime}$, we have a simple test, denoted Incl (x, $\mathrm{x}^{\prime}$ ), to decide if $D_{x}$ contains $D_{x^{\prime}}$ (we just compare segment extremities). Let us denote by $\left[l_{y}, r_{y}\right]$ the interval given by a disk $D_{y}$. We consider the list $\mathcal{L}$ of parabolas sorted according to the left extremity of the segments. If some parabolas have got the same left extremity coordinate, we sort such parabolas according to the right extremity position (see figure 4-(right)). If $n$ denotes the size of the column $j$ in the image, the list $\mathcal{L}$ can be computed in $O(n)$ (we store the extremities in two arrays of size $n$ during the scan of the parabolas). If two segments are identical, we remove one of them and we label the other one with a flag "double" (see definition 4). Using $\mathcal{L}$, we have a simple algorithm presented in figure 4 - $(b)$ to remove from the set $\left\{\mathcal{F}_{x}(i)\right\}$ all points that do not belong to the discrete medial axis. In this algorithm, we scan the parabolas according to the $\mathcal{L}$ order and we test the inclusion of two consecutive parabolas in a greedy process. Hence, the computational cost of this filtering algorithm is $O(n)$. The resulting set of parabolas is stored in the array $s$ and the correctness of this algorithm is given by the proposition:

Proposition 2. The associated disk of a parabola is maximal if and only if the parabola belongs to $s$ at the end of the filtering process.

Proof. First of all, if the list $\mathcal{L}$ is reduced to one parabola, the associated disk is maximal and it belongs to $s$. We prove the proposition by induction. Let us consider the step $k(k \geq 1)$ in the algorithm 4 - $(b)$. We suppose that, at this point, $s$ contains the maximal disk of the parabolas in $\{\mathcal{L}(i)\}_{0 \leq i \leq k}$ and we consider the disk $[u, v]$ of the parabola $\mathcal{L}(k+1)$. Note that the order of parabola in $s$ is the same as the order of parabola in $\mathcal{L}$. We denote by $[m, n]$ the segment associated to $s[q]$ (last inserted parabola in $s$ ). If the test $\operatorname{Incl}(s[q], \mathcal{L}(k+1))$ is true, the segment $[m, n]$ contains the segment $[u, v]$, and so $\mathcal{L}(k+1)$ is not maximal and this parabola is not inserted in $s$. If we suppose that the inclusion test fails, $\mathcal{L}(k+1)$ is inserted in $s$. First of all, the segment $[u, v]$ cannot contain a segment in $s$. Indeed, by definition of $\mathcal{L}$, if a parabola $x$ is before the parabola $x^{\prime}$ in $\mathcal{L}$, then the segment associated to $x^{\prime}$ cannot contain the segment associated to $x$. Hence $\mathcal{L}(k+1)$ does not change the maximal property of the segments in $s$. To complete the proof, we show that if the test fails, no segment in $s$ contains the segment $[u, v]$. Let us consider a segment $[a, b]$ in $s$ such that $[a, b]$ contains 
$[u, v]$ and such that the segment $[a, b]$ is not associated to $s[q]$. So, we have $b \geq v$ and $a \leq u$. If the inclusion test fails between $[u, v]$ and $[m, n]$, then $v>n$ (we have $u \geq m$ by construction of $\mathcal{L}$ ). Hence, we have $b>v$. This leads to the contradiction that $[a, b]$ contains $[m, n]$ because the segments in $s$ are supposed to be maximal. Finally, it is sufficient to consider the inclusion test between $\mathcal{L}(k+1)$ and the last inserted parabola in $s$ to construct the set of maximal disks from the set $\{\mathcal{L}(i)\}_{0 \leq i \leq k+1}$.

In higher dimensions, we apply this filtering process in each dimension and we define the reduced medial axis as follows:

Definition 4 (Reduced Medial Axis). Let $P$ be a binary shape in dimension $d$ and $Q$ the SDT of $P$. We consider Sk the Satio and Toriwaki's skeleton of $P$. The reduced medial axis (RMA for short) is the set of points $(i, j)$ such that there exists at least one row in one of the $d$ dimensions in which the parabola associated to $\left(i, j, q_{i j}\right)$ is preserved and not labeled "double" during the onedimensional filtering process.

Theorem 1. Let $P$ be a binary shape in dimension $d$, the $R M A$ is a subset of the discrete medial axis of the shape, it has the reversibility property and the $R M A$ extraction is in $O\left(n^{d}\right)$.

Proof. According to the corollary 1, $S k$ is a subset of the continuous medial axis of the shape. Let us consider a discrete ball $B$, we prove that if $B$ is preserved at the end of the filtering process, then $B$ belongs to the discrete medial axis. If we suppose that $B$ is not maximal in the discrete case, there exists another ball $B^{\prime}$ such that $B^{\prime}$ contains $B$. During the filtering process, in each dimension, the segments associated to $B$ will either be removed or labeled "double" because they are contained in $B^{\prime}$ segments. Hence, the ball $B$ will be removed from $S k$. Finally, all resulting balls are maximal in the discrete plane. Furthermore, since the parabola removal process between two parabolas maintains the reversibility property, the final result allows us to reconstruct the shape. Concerning the computational cost, the $S k$ computation is done in $O\left(n^{d}\right)$ and for each row in each dimension, the one-dimensional filtering process computational cost is linear in the number of parabolas in the row. Hence the global cost of the filtering is linear in the number of points in $P$, which is optimal for the problem.

\subsection{Results and Generalizations}

In this section we present some results of both the REDT and skeleton extraction algorithms in dimension 2 and 3. The figure 5 presents results on several 2-D and 3-D shapes. Note that the EDT of images is computed using the Hirata's algorithm [7].

Due to the one-dimensional decomposition process of the REDT and skeleton extraction algorithms, several generalizations that have been proposed for EDT algorithm can be done. For example, the same algorithms can be used for $d$ dimensional elongated grids (different scale factors between axis). We just have to insert those scale factors in the process without changing the algorithms [18]. 

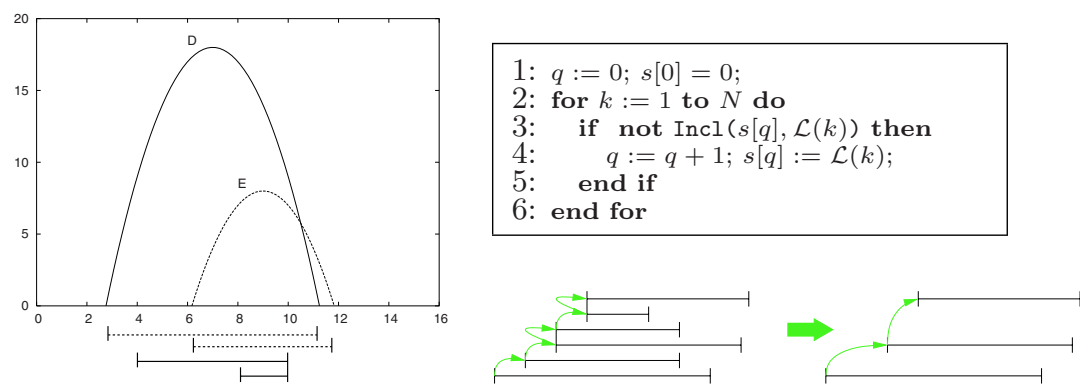

Fig. 4. An illustration of the difference between the skeleton $S k$ and the discrete medial axis (left): dashed segments indicate Euclidean balls $\left\{\mathcal{D}_{x}\right\}$ and plain segments the discrete balls $\left\{D_{x}\right\}$. Right: Pseudo-code for the filtering process of $S k$ points and illustration of the algorithm (arrows indicate the order in $\mathcal{L}$ or in $s$ ).

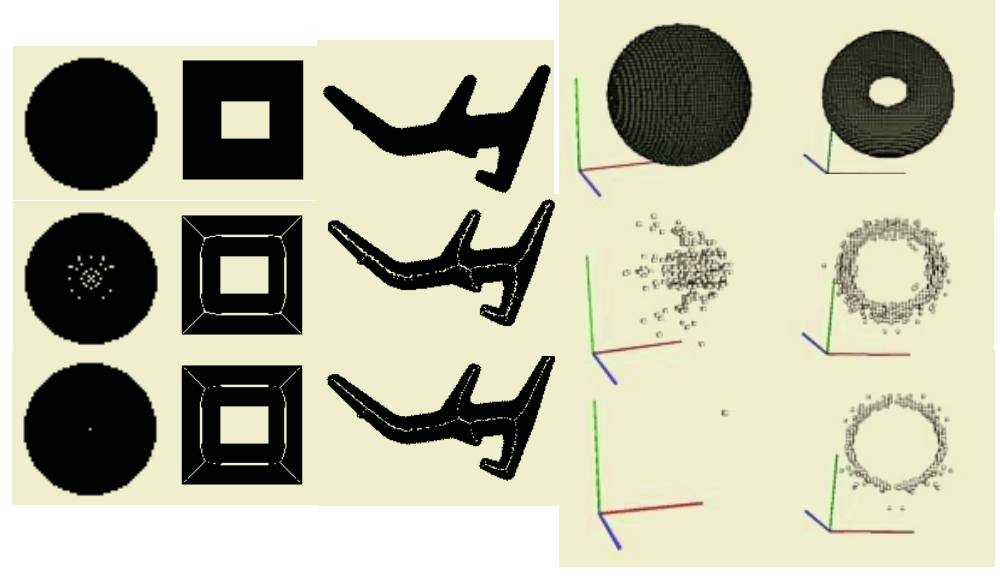

Fig. 5. Results of skeleton extraction on 2-D and 3-D images: first row the input binary shapes, second row the $S k$ skeleton extraction and last row RMA extraction results.

\section{Conclusion}

In this article, we first have optimized the REDT computation algorithm and have obtained a computation cost in $O\left(n^{d}\right)$ for a $d$-dimensional image which is optimal in time ( $n^{d}$ is the total number of grid points). Then, we have presented a $d$-dimensional reversible RMA extraction algorithm in $O\left(n^{d}\right)$. We have shown that the proposed RMA is a subset of the classical medial axis of the shape. In future works, we expect further optimizations of the RMA extraction process to reduce again the number of points. The final goal of this optimization should be to compute the optimal reversible skeleton of a shape (in the sense of having minimal number of points, see $[3,11]$ for related papers). Furthermore, we would like to illustrate the $d$-dimensional algorithms on real data in higher dimensions. 


\section{References}

1. H. Blum. A transformation for extracting descriptors of shape. In Models for the Perception of Speech and Visual Forms, pages 362-380. MIT Press, 1967.

2. G. Borgefors. Distance transformations in digital images. Computer Vision, Graphics, and Image Processing, 34(3):344-371, June 1986.

3. G. Borgefors and I. Nyström. Efficient shape representation by minimizing the set of centers of maximal discs/spheres. Pattern Recognition Letters, 18:465-472, 1997.

4. D. Coeurjolly. Algorithmique et géométrie discrète pour la caractérisation des courbes et des surfaces. PhD thesis, Université Lumière Lyon 2, Bron, Laboratoire ERIC, dec 2002.

5. P. E. Danielsson. Euclidean distance mapping. CGIP, 14:227-248, 1980.

6. G. Sanniti di Baja. Well-shaped, stable, and reversible skeletons from the $(3,4)$ distance transform. J. Visual Communication and Image Representation, 5:107$115,1994$.

7. T. Hirata. A unified linear-time algorithm for computing distance maps. Information Processing Letters, 58(3):129-133, May 1996.

8. C.R. Maurer Jr., V. Raghavan, and R. Qi. A linear time algorithm for computing the euclidean distance transform in arbitrary dimensions. In Information Processing in Medical Imaging, pages 358-364, 2001.

9. A. Meijster, J.B.T.M. Roerdink, and W. H. Hesselink. A general algorithm for computing distance transforms in linear time. In Mathematical Morphology and its Applications to Image and Signal Processing, pages 331-340. Kluwer, 2000.

10. U. Montanari. Continuous skeletons from digitized images. Journal of the Association for Computing Machinery, 16(4):534-549, oct 1969.

11. F. Nilsson and P.-E. Danielsson. Finding the minimal set of maximum disks for binary objects. Graphical models and image processing, 59(1):55-60, January 1997.

12. I. Ragnemalm. Contour processing distance transforms, pages 204-211. World Scientific, 1990.

13. I. Ragnemalm. The Euclidean Distance Transform. PhD thesis, Linköping University, Linköping, Sweden, 1993.

14. E. Remy and E. Thiel. Optimizing 3D chamfer masks with norm constraints. In Int. Workshop on Combinatorial Image Analysis, pages 39-56, Caen, July 2000.

15. A. Rosenfeld and J. L. Pfaltz. Sequential operations in digital picture processing. Journal of the ACM, 13(4):471-494, October 1966.

16. A. Rosenfeld and J. L. Pfalz. Distance functions on digital pictures. Pattern Recognition, 1:33-61, 1968.

17. T. Saito and J. I. Toriwaki. New algorithms for Euclidean distance transformations of an $n$-dimensional digitized picture with applications. Pattern Recognition, 27:1551-1565, 1994.

18. T. Saito and J.-I. Toriwaki. Reverse distance transformation and skeletons based upon the euclidean metric for $n$-dimensionnal digital pictures. IECE Trans. Inf. E Syst., E77-D(9):1005-1016, sept. 1994.

19. E. Thiel. Géométrie des distances de chanfrein. Habilitation à Diriger des Recherches, Université de la Méditerranée, Aix-Marseille 2, Déc 2001. 